\title{
MODELS FOR THE ESTIMATION OF THERMODYNAMIC PROPERTIES OF LAYERED DOUBLE HYDROXIDES: APPLICATION TO THE STUDY OF THEIR ANION EXCHANGE CHARACTERISTICS
}

\author{
Juan J. Bravo-Suárez ${ }^{\#}$ \\ Centro de Investigaciones en Catálisis, Escuela de Ingeniería Química, Universidad Industrial de Santander, A.A. 678 Bucaramanga, \\ Colombia and Environmental Catalysis and Nanomaterials Laboratory, Department of Chemical Engineering, Virginia Polytechnic \\ Institute and State University, Blacksburg, Virginia 24061-0211 \\ Edgar A. Páez-Mozo \\ Centro de Investigaciones en Catálisis, Escuela de Química, Universidad Industrial de Santander, A.A. 678 Bucaramanga, Colombia \\ S. Ted Oyama* \\ Environmental Catalysis and Nanomaterials Laboratory, Department of Chemical Engineering, Virginia Polytechnic Institute and \\ State University, Blacksburg, Virginia 24061-0211
}

Recebido em 14/8/03; aceito em 7/11/03; publicado na web em 27/05/04

\begin{abstract}
Several models for the estimation of thermodynamic properties of layered double hydroxides (LDHs) are presented. The predicted thermodynamic quantities calculated by the proposed models agree with experimental thermodynamic data. A thermodynamic study of the anion exchange process on LDHs is also made using the described models. Tables for the prediction of monovalent anion exchange selectivities on LDHs are provided. Reasonable agreement is found between the predicted and the experimental monovalent anion exchange selectivities.
\end{abstract}

Keywords: layered double hydroxides; thermodynamic properties; model.

\section{INTRODUCTION}

There is presently a great interest in layered double hydroxide materials due to their well-known industrial applications as catalysts and catalyst supports ${ }^{1-3}$, adsorbents ${ }^{4}$, anion exchangers ${ }^{5}$, flame retardants $^{6}$, polymer stabilizers ${ }^{7}$ and antacids ${ }^{8}$. Attention to these materials is growing because of many new emerging applications, such as in thin films ${ }^{9}$, conducting materials ${ }^{10}$, corrosion protectors ${ }^{11}$, electrodes ${ }^{12}$ and as hosts for the controlled release of chemicals ${ }^{13,14}$.

Layered double hydroxides can be represented by the general formula: $\left[\mathrm{M}_{1-\mathrm{x}}^{2+} \mathrm{M}_{\mathrm{x}}^{3+}(\mathrm{OH})_{2}\right]^{\mathrm{x}+}\left(\mathrm{A}_{\mathrm{x} / \mathrm{n}}^{\mathrm{n}-}\right) \cdot \mathrm{mH}_{2} \mathrm{O}$, where $\mathrm{M}^{2+}$ and $\mathrm{M}^{3+}$ can be any divalent and trivalent metal ion whose ionic radius is similar to that of $\mathrm{Mg}^{2+}, \mathrm{A}^{\mathrm{n}-}$ can be any compensating anion, $\mathrm{m}$ is the amount of water present in the interlamellar region and $\mathrm{x}$ is the ratio $\mathrm{M}^{3+} /\left(\mathrm{M}^{2+}+\mathrm{M}^{3+}\right)^{1}$. The most common natural $\mathrm{LDH}$ is the $\mathrm{Mg}-\mathrm{Al}$ hydrotalcite whose structure is based on that of brucite, $\left[\mathrm{Mg}(\mathrm{OH})_{2}\right]$, where $\mathrm{Mg}^{2+}$ is octahedrally coordinated to six hydroxyl groups. In brucite the octahedra share edges to form bidimensional layers stacked one on top of the other which are held together by weak interactions through hydrogen atoms ${ }^{15}$. When some of these $\mathrm{Mg}^{2+}$ ions are replaced by cations of higher charge, as occurs in the $\mathrm{Mg}$ - $\mathrm{Al}$ compound by the introduction of $\mathrm{Al}^{3+}$, positive charge is formed in the brucite-like layers. This positive charge is neutralized by compensating anions in the interlamellar region and, in this manner, the hydrotalcite structure is formed. Some water molecules are also found in the interlamellar region. Commonly, cations in octahedral coordination occupy random positions in the brucite-like layers ${ }^{16}$. The anion and the water molecules are randomly located in the interlayer region and are labile, being free to move by breaking bonds to the layers and forming new ones ${ }^{17}$.

*e-mail: oyama@vt.edu

\# Current address: Research Institute for Innovation in Sustainable Chemistry, National Institute of Advanced Industrial Science and Technology (AIST), 16-1 Onogawa, Tsukuba 305-8569, Japan.
Even though literature on LDH materials is abundant ${ }^{18}$, thermodynamic studies are limited. This fact may be explained by the lack of necessary thermodynamic data and the great amount of experimental work needed to cover the wide range of compositional variations of LDHs. Tools for the estimation of the thermodynamic properties of LDHs in the literature are scarce. Recently, Allada et al. ${ }^{19}$ estimated the thermodynamic properties of $\left[\mathrm{M}_{1-\mathrm{x}}^{2+} \mathrm{Al}_{\mathrm{x}}^{3+}(\mathrm{OH})_{2}\right]^{\mathrm{x}+}$ $\left(\mathrm{CO}_{3}\right)_{\mathrm{x} / 2} \cdot \mathrm{mH}_{2} \mathrm{O} \mathrm{LDH}$ compounds from $\mathrm{M}^{2+}(\mathrm{OH})_{2}, \mathrm{Al}(\mathrm{OH})_{3}$ and the carbonate form of the divalent metal. They found good results compared with the experimental thermodynamic quantities of some carbonate LDHs. We have recently extended the application of this model to $\left[\mathrm{M}_{1-\mathrm{x}}^{2+} \mathrm{M}_{\mathrm{x}}^{3+}(\mathrm{OH})_{2}\right]^{\mathrm{x}+}\left(\mathrm{A}^{\mathrm{n}-}\right)_{\mathrm{x} / \mathrm{n}} \mathrm{LDHs}^{20}$. We also found good agreement between estimated thermodynamic quantities and published experimental results on LDH solubility and the synthesis of LDHs by hydrothermal-reconstruction and coprecipitation methods.

On the basis of the LDH structure, we present in this study new mixture models for the estimation of thermodynamic properties of LDHs. A thermodynamic study of the anion exchange properties of LDH compounds, using the different mixture models, is also given.

\section{SINGLE MODELS}

The general idea in a mixture model for a LDH is basically treating the $\mathrm{LDH}$ as a mixture of structurally similar simple compounds. If we consider one of the layer-upon-layer arrangements of brucite-like layers, for example, when an anion (A) is in a trigonal prismatic environment ${ }^{21}$ of $\mathrm{OH}$ groups for a dehydrated phase, as seen in Figure 1, several combinations of simple compounds in the LDH structure are found: Model 0: a combination of the compounds $\mathrm{M}^{2+}(\mathrm{OH})_{2}, \mathrm{M}^{3+}(\mathrm{OH})_{3}$ and the ion $\left(\mathrm{A}^{\mathrm{n}-}\right)$

Model 1: a combination of the compounds $\mathrm{M}^{2+}(\mathrm{OH})_{2}, \mathrm{M}^{3+}(\mathrm{OH})_{3}$ and $\mathrm{H}_{\mathrm{n}}\left(\mathrm{A}^{\mathrm{n}-}\right)$

Model 2: a combination of the compounds $\mathrm{M}^{2+}(\mathrm{OH})_{2}, \mathrm{M}^{3+}(\mathrm{OH})_{3}$ and $\mathrm{M}^{2+}\left(\mathrm{A}^{\mathrm{n}-}\right)$ /n 


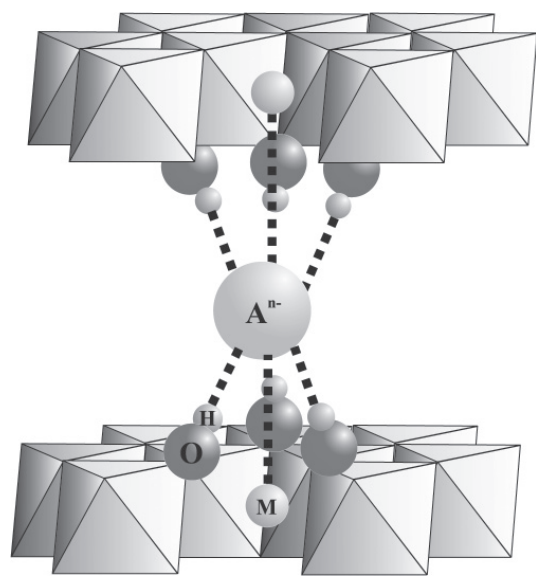

Figure 1. Structure of a LDH with stacking of brucite-like layers with the anion in a prismatic environment of $\mathrm{OH}$ groups. $\mathrm{A}=$ compensating anion, $O=$ oxygen, $H=$ hydrogen and $M=$ any divalent or trivalent cation. The octahedron around cation $M$ is not shown for the sake of clarity

Model 3: a combination of the compounds $\mathrm{M}^{2+}(\mathrm{OH})_{2}, \mathrm{M}^{3+}(\mathrm{OH})_{3}$ and $\mathrm{M}_{\mathrm{n}}^{3+}\left(\mathrm{A}^{\mathrm{n}-}\right)_{3}$

where $\mathrm{n}=1,2$

After a molar balance of the constituting compounds and the final $\mathrm{LDH}$, the following equations are obtained for the different models.

Model 0 [f $\left.\mathbf{f}_{\mathbf{0}}(\mathrm{LDH})\right]$ :

$(1-\mathrm{x}) \mathrm{M}^{2+}(\mathrm{OH})_{2}(\mathrm{~s})+(\mathrm{x}) \mathrm{M}^{3+}(\mathrm{OH})_{3}(\mathrm{~s})+(\mathrm{x} / \mathrm{n}) \mathrm{A}^{\mathrm{n}-}(\mathrm{aq})=$ $\left[\mathrm{M}_{1-\mathrm{x}}^{2+} \mathrm{M}_{\mathrm{x}}^{3+}(\mathrm{OH})_{2}\right]\left(\mathrm{A}^{\mathrm{n}-}\right)_{\mathrm{x} / \mathrm{n}}(\mathrm{s})+\mathrm{xOH}^{-}(\mathrm{aq})$

Model 1 [ $\left.\mathbf{f}_{1}(\mathbf{L D H})\right]$ :

$(1-\mathrm{x}) \mathrm{M}^{2+}(\mathrm{OH})_{2}(\mathrm{~s})+(\mathrm{x}) \mathrm{M}^{3+}(\mathrm{OH})_{3}(\mathrm{~s})+(\mathrm{x} / \mathrm{n}) \mathrm{H}_{\mathrm{n}}\left(\mathrm{A}^{\mathrm{n}-}\right)(\mathrm{aq})=$ $\left[\mathrm{M}_{1-\mathrm{x}}^{2+} \mathrm{M}_{\mathrm{x}}^{3+}(\mathrm{OH})_{2}\right]\left(\mathrm{A}^{\mathrm{n}-}\right)_{\mathrm{x} / \mathrm{n}}(\mathrm{s})+\mathrm{xH}_{2} \mathrm{O}(\mathrm{aq})$

Model 2 [f $\mathbf{f}(\mathbf{L D H})]$ :

$(1-3 \mathrm{x} / 2) \mathrm{M}^{2+}(\mathrm{OH})_{2}(\mathrm{~s})+(\mathrm{x}) \mathrm{M}^{3+}(\mathrm{OH})_{3}(\mathrm{~s})+(\mathrm{x} / 2) \mathrm{M}^{2+}\left(\mathrm{A}^{\mathrm{n}-}\right)_{2 / \mathrm{n}}(\mathrm{s})=$

$$
\left[\mathrm{M}_{1-\mathrm{x}}^{2+} \mathrm{M}_{\mathrm{x}}^{3+}(\mathrm{OH})_{2}\right]\left(\mathrm{A}^{\mathrm{n}-}\right)_{\mathrm{x} / \mathrm{n}}(\mathrm{s})
$$

Model 3 [ $\left.\mathbf{f}_{\mathbf{3}}(\mathrm{LDH})\right]$ :

$(1-\mathrm{x}) \mathrm{M}^{2+}(\mathrm{OH})_{2}(\mathrm{~s})+(2 \mathrm{x} / 3) \mathrm{M}^{3+}(\mathrm{OH})_{3}(\mathrm{~s})+(\mathrm{x} / 3 \mathrm{n}) \mathrm{M}^{3}{ }_{\mathrm{n}}^{+}\left(\mathrm{A}^{\mathrm{n}-}\right)_{3}(\mathrm{~s})=$

$$
\left[\mathrm{M}_{1-\mathrm{x}}^{2+} \mathrm{M}_{\mathrm{x}}^{3+}(\mathrm{OH})_{2}\right]\left(\mathrm{A}^{\mathrm{n}-}\right)_{\mathrm{x} / \mathrm{n}}(\mathrm{s})
$$

Model 2 is similar to the mixture model proposed by Allada et al. ${ }^{19}$.
Equations 1, 2, 3 and 4 can be used for the calculations of thermodynamic properties of LDH compounds. As an example, standard Gibbs free energies of formation of LDHs are calculated. Model 0:

$$
\begin{aligned}
\Delta_{\mathrm{f}, \mathrm{M} 0} \mathrm{G}_{\mathrm{m}}^{\mathrm{o}} & \{\mathrm{T}, \mathrm{LDH}\}=(1-\mathrm{x}) \Delta_{\mathrm{f}} \mathrm{G}_{\mathrm{m}}^{\mathrm{o}}\left\{\mathrm{T}, \mathrm{M}^{2+}(\mathrm{OH})_{2}\right\} \\
& +(\mathrm{x}) \Delta_{\mathrm{f}} \mathrm{G}_{\mathrm{m}}^{\mathrm{o}}\left\{\mathrm{T}, \mathrm{M}^{3+}(\mathrm{OH})_{3}\right\}+(\mathrm{x} / \mathrm{n}) \Delta_{\mathrm{f}} \mathrm{G}_{\mathrm{m}}^{\mathrm{o}}\left\{\mathrm{T}, \mathrm{A}^{\mathrm{n}-}\right\} \\
& -(\mathrm{x}) \Delta_{\mathrm{f}} \mathrm{G}_{\mathrm{m}}^{\mathrm{o}}\left\{\mathrm{T}, \mathrm{OH}^{-}\right\}
\end{aligned}
$$

Model 1:

$$
\begin{aligned}
\Delta_{\mathrm{f}, \mathrm{M} 1} \mathrm{G}_{\mathrm{m}}^{\mathrm{o}} & \{\mathrm{T}, \mathrm{LDH}\}=(1-\mathrm{x}) \Delta_{\mathrm{f}} \mathrm{G}_{\mathrm{m}}^{\mathrm{o}}\left\{\mathrm{T}, \mathrm{M}^{2+}(\mathrm{OH})_{2}\right\} \\
& +(\mathrm{x}) \Delta_{\mathrm{f}} \mathrm{G}_{\mathrm{m}}^{\mathrm{o}}\left\{\mathrm{T}, \mathrm{M}^{3+}(\mathrm{OH})_{3}\right\}+(\mathrm{x} / \mathrm{n}) \Delta_{\mathrm{f}} \mathrm{G}_{\mathrm{m}}^{\mathrm{o}}\left\{\mathrm{T}, \mathrm{H}_{\mathrm{n}}\left(\mathrm{A}^{\mathrm{n}-}\right)\right\} \\
& -(\mathrm{x}) \Delta_{\mathrm{f}} \mathrm{G}_{\mathrm{m}}^{\mathrm{o}}\left\{\mathrm{T}, \mathrm{H}_{2} \mathrm{O}\right\}
\end{aligned}
$$

Model 2:

$\Delta_{\mathrm{f}, \mathrm{M} 2} \mathrm{G}_{\mathrm{m}}^{\mathrm{o}}\{\mathrm{T}, \mathrm{LDH}\}=(1-3 \mathrm{x} / 2) \Delta_{\mathrm{f}} \mathrm{G}_{\mathrm{m}}^{\mathrm{o}}\left\{\mathrm{T}, \mathrm{M}^{2+}(\mathrm{OH})_{2}\right\}$

$$
+(\mathrm{x}) \Delta_{\mathrm{f}} \mathrm{G}_{\mathrm{m}}^{\mathrm{o}}\left\{\mathrm{T}, \mathrm{M}^{3+}(\mathrm{OH})_{3}\right\}+(\mathrm{x} / 2) \Delta_{\mathrm{f}} \mathrm{G}_{\mathrm{m}}^{\mathrm{o}}\left\{\mathrm{T}, \mathrm{M}^{2+}\left(\mathrm{A}^{\mathrm{n}-}\right)_{2 / \mathrm{n}}\right\}
$$

Model 3:

$\Delta_{\mathrm{f}, \mathrm{M} 3} \mathrm{G}_{\mathrm{m}}^{\mathrm{o}}\{\mathrm{T}, \mathrm{LDH}\}=(1-\mathrm{x}) \Delta_{\mathrm{f}} \mathrm{G}_{\mathrm{m}}^{\mathrm{o}}\left\{\mathrm{T}, \mathrm{M}^{2+}(\mathrm{OH})_{2}\right\}$

$$
+(2 \mathrm{x} / 3) \Delta_{\mathrm{f}} \mathrm{G}_{\mathrm{m}}^{\mathrm{o}}\left\{\mathrm{T}, \mathrm{M}^{3+}(\mathrm{OH})_{3}\right\}+(\mathrm{x} / 3 \mathrm{n}) \Delta_{\mathrm{f}} \mathrm{G}_{\mathrm{m}}^{\mathrm{o}}\left\{\mathrm{M}_{\mathrm{n}}^{3+}\left(\mathrm{A}^{\mathrm{n}-}\right)_{3}\right\}
$$

Examples of standard Gibbs free energies of formation for typical LDHs, using models $0,1,2$ and 3, are shown in Table 1. For the calculations Equations 5, 6, 7 and 8 were used. For example, the standard Gibbs free energy of formation for the $\left[\mathrm{Zn}_{0.75} \mathrm{Al}_{0.25}(\mathrm{OH})_{2}\right]\left(\mathrm{NO}_{3}\right)_{0.25} \mathrm{LDH}$ calculated by model 1 is:

$$
\begin{aligned}
\Delta_{\mathrm{f}, \mathrm{M} 1} \mathrm{G}_{\mathrm{m}}^{\mathrm{o}} & \left\{298.15 \mathrm{~K}, \mathrm{ZnAl}-\mathrm{NO}_{3}\right\}=(1-\mathrm{x}) \Delta_{\mathrm{f}} \mathrm{G}_{\mathrm{m}}^{\mathrm{o}}\left\{298.15 \mathrm{~K}, \mathrm{Zn}(\mathrm{OH})_{2}\right\} \\
& +(\mathrm{x}) \Delta_{\mathrm{f}} \mathrm{G}_{\mathrm{m}}^{\mathrm{o}}\left\{298.15 \mathrm{~K}, \mathrm{Al}(\mathrm{OH})_{3}\right\} \\
& +(\mathrm{x}) \Delta_{\mathrm{f}} \mathrm{G}_{\mathrm{m}}^{\mathrm{o}}\left\{298.15 \mathrm{~K}, \mathrm{HNO}_{3}\right\}-(\mathrm{x}) \Delta_{\mathrm{f}} \mathrm{G}_{\mathrm{m}}^{\mathrm{o}}\left\{298.15 \mathrm{~K}, \mathrm{H}_{2} \mathrm{O}\right\}
\end{aligned}
$$

\begin{tabular}{|c|c|c|c|c|c|}
\hline \multirow[b]{2}{*}{$\mathrm{LDH}$} & \multicolumn{5}{|c|}{$\Delta_{\mathrm{f}} \mathrm{G}_{\mathrm{m}}^{o}\{298.15 \mathrm{~K}, \mathrm{LDH}\}\left(\mathrm{kJ} \mathrm{mol}^{-1}\right)$} \\
\hline & Model 0 & Model 1 & Model 2 & Model 3 & General Model \\
\hline$\left[\mathrm{Mg}_{0.75} \mathrm{Al}_{0.25}(\mathrm{OH})_{2}\right](\mathrm{Cl})_{0.25}$ & -907.9 & -887.8 & -884.2 & -879.5 & -897.1 \\
\hline$\left[\mathrm{Mg}_{0.75} \mathrm{Al}_{0.25}(\mathrm{OH})_{2}\right]\left(\mathrm{NO}_{3}\right)_{0.25}$ & -902.9 & -882.9 & -883.8 & -882.9 & -893.0 \\
\hline$\left[\mathrm{Ni}_{0.75} \mathrm{Al}_{0.25}(\mathrm{OH})_{2}\right](\mathrm{Cl})_{0.25}$ & -614.1 & -594.0 & -597.8 & -585.7 & -604.2 \\
\hline$\left[\mathrm{Ni}_{0.75} \mathrm{Al}_{0.25}(\mathrm{OH})_{2}\right]\left(\mathrm{NO}_{3}\right)_{0.25}$ & -609.1 & -589.1 & -594.9 & -589.1 & -599.8 \\
\hline$\left[\mathrm{Zn}_{0.75} \mathrm{Al}_{0.25}(\mathrm{OH})_{2}\right](\mathrm{Cl})_{0.25}$ & -698.6 & -678.5 & -682.2 & -670.1 & -688.7 \\
\hline$\left[\mathrm{Zn}_{0.75} \mathrm{Al}_{0.25}(\mathrm{OH})_{2}\right]\left(\mathrm{NO}_{3}\right)_{0.25}$ & -693.6 & -673.6 & -673.1 & -673.6 & -683.5 \\
\hline$\left[\mathrm{Fe}_{0.67} \mathrm{Fe}_{0.33}(\mathrm{OH})_{2}\right]\left(\mathrm{SO}_{4}\right)_{0.17}$ & -632.5 & -606.0 & -616.6 & -630.5 & -621.8 \\
\hline$\left[\mathrm{Fe}_{0.75} \mathrm{Fe}_{0.25}(\mathrm{OH})_{2}\right](\mathrm{Cl})_{0.25}$ & -535.2 & -515.2 & -519.2 & -510.2 & -525.5 \\
\hline$\left[\mathrm{Fe}_{0.67} \mathrm{Fe}_{0.33}(\mathrm{OH})_{2}\right]\left(\mathrm{CO}_{3}\right)_{0.17}$ & -596.5 & -585.7 & -592.6 & $\mathrm{x}$ & $\mathrm{x}$ \\
\hline
\end{tabular}

$$
\begin{gathered}
\Delta_{\mathrm{f}, \mathrm{Ml}} \mathrm{G}_{\mathrm{m}}^{\mathrm{o}}\left\{298.15 \mathrm{~K}, \mathrm{ZnAl}-\mathrm{NO}_{3}\right\}=0.75(-554.5)+0.25(-1156.9) \\
+0.25(-111.3)-0.25(-237.2)=-673.6 \mathrm{~kJ} \mathrm{~mol}^{-1}
\end{gathered}
$$

Other entries in Table 1 are calculated in a similar fashion. The standard Gibbs free energies of formation for all the compounds were gathered from different well-known compilations of thermodynamic data: Naumov et al. ${ }^{22}$, Karapet'yants et al. ${ }^{23}$ and Perry et al. ${ }^{24}$.

Formulas for the standard enthalpies of formation can also be derived. These formulas are similar to Equations 5, 6, 7 and 8 . Examples of the calculations for the standard enthalpies of formation for some LDHs are given in Table 2. For instance, the standard enthalpy of formation for the $\left[\mathrm{Co}_{0.68} \mathrm{Al}_{0.32}(\mathrm{OH})_{2}\right]\left(\mathrm{CO}_{3}\right)_{0.17} \cdot 0.80 \mathrm{H}_{2} \mathrm{O}$ $\mathrm{LDH}$ calculated by model 0 is:

Table 1. Standard Gibbs free energies of formation for some LDHs

$\mathrm{x}=$ not enough data available 
Table 2. Standard enthalpies of formation for some LDHs

\begin{tabular}{lccccc}
\hline & \multicolumn{4}{c}{$\Delta_{\mathrm{f}} \mathrm{H}_{\mathrm{m}}^{\mathrm{o}}\{298.15 \mathrm{~K}, \mathrm{LDH}\}\left(\mathrm{kJ} \mathrm{mol}{ }^{-1}\right)$} \\
\cline { 2 - 6 } $\mathrm{LDH}$ & Model 0 & Model 1 & Model 2 & Model 3 & General Model \\
\hline$\left[\mathrm{Co}_{0.68} \mathrm{Al}_{0.32}(\mathrm{OH})_{2}\right]\left(\mathrm{CO}_{3}\right)_{0.17} \cdot 0.80 \mathrm{H}_{2} \mathrm{O}$ & -1041.7 & -1027.4 & -1039.1 & $\mathrm{x}$ & $\mathrm{x}$ \\
{$\left[\mathrm{Co}_{0.76} \mathrm{Al}_{0.24}(\mathrm{OH})_{2}\right]\left(\mathrm{CO}_{3}\right)_{0.12} \cdot 0.81 \mathrm{H}_{2} \mathrm{O}$} & -984.0 & -973.1 & -982.0 & $\mathrm{x}$ & $\mathrm{x}$ \\
\hline
\end{tabular}

$\mathrm{x}=$ not enough data available

$$
\begin{aligned}
\Delta_{\mathrm{f}, \mathrm{M} 0} \mathrm{H}_{\mathrm{m}}^{\circ} & \left\{298.15 \mathrm{~K}, \mathrm{CoAl}-\mathrm{CO}_{3} \cdot \mathrm{mH}_{2} \mathrm{O}\right\}= \\
& (1-\mathrm{x}) \Delta_{\mathrm{f}} \mathrm{H}_{\mathrm{m}}{ }^{\circ}\left\{298.15 \mathrm{~K}, \mathrm{Co}(\mathrm{OH})_{2}\right\} \\
& +(\mathrm{x}) \Delta_{\mathrm{f}} \mathrm{H}_{\mathrm{m}}^{\circ}\left\{298.15 \mathrm{~K}, \mathrm{Al}(\mathrm{OH})_{3}\right\}+(\mathrm{x} / 2) \Delta_{\mathrm{f}} \mathrm{H}_{\mathrm{m}}^{\mathrm{o}}\left\{298.15 \mathrm{~K}, \mathrm{CO}_{3}{ }^{2-}\right\} \\
& -(\mathrm{x}) \Delta_{\mathrm{f}} \mathrm{H}_{\mathrm{m}}^{\mathrm{o}}\left\{298.15 \mathrm{~K}, \mathrm{OH}^{-}\right\}+(\mathrm{m}) \Delta_{\mathrm{f}} \mathrm{H}_{\mathrm{m}}^{\mathrm{o}}\left\{298.15 \mathrm{~K}, \mathrm{H}_{2} \mathrm{O}\right\}
\end{aligned}
$$$$
\Delta_{\mathrm{f}, \mathrm{M} 0} \mathrm{H}_{\mathrm{m}}^{\circ}\left\{298.15 \mathrm{~K}, \mathrm{CoAl}-\mathrm{CO}_{3} \cdot \mathrm{mH}_{2} \mathrm{O}\right\}=0.68(-554.7)
$$$$
+0.32(-1293.3)+(0.25 / 2)(-677.1)-0.25(-230.0)
$$$$
+0.80(-285.8)=-1041.7 \mathrm{~kJ} \mathrm{~mol}^{-1}
$$

The required standard enthalpies of formation for the compounds used in model 2 were taken from Allada et al. ${ }^{19}$. Additional thermodynamic data were gathered from well-known compilations ${ }^{22-24}$.

\section{GENERAL MODEL}

As seen from Table 1, all of the single models give similar results; however, the selection of just one model for the estimation of LDH thermodynamic properties would require proofs at an atomistic level. Several works of molecular dynamic modeling of LDHs have been carried out recently ${ }^{25-29}$. Although these molecular modeling studies may be useful when choosing among the single models, this approach would be only applicable to specific cases. Again, numerous studies would be necessary to cover all the possible combinations of cations, anions and the range of different compositions and even some of the structural differences among LDHs. Considering all these factors, a general model involving all of the single models is developed.

Starting from a LDH with the formula: $\left[\mathrm{M}_{1-\mathrm{x}}^{2+} \mathrm{M}_{\mathrm{x}}^{3+}(\mathrm{OH})_{2}\right]^{\mathrm{x}+}\left(\mathrm{A}^{\mathrm{n}-}\right)_{\mathrm{x} / \mathrm{n}}$, the number of cations $\left(\mathrm{NC}_{\mathrm{i}}\right.$, where i refers to the model number) that may interact with $\mathrm{A}^{\mathrm{n}-}$ are:

$\mathrm{NC}_{0} \quad=0$

$\mathrm{NC}_{1}\left(\mathrm{H}^{+}\right)=2$

$\mathrm{NC}_{2}\left(\mathrm{M}^{2+}\right)=1-\mathrm{x}$

$\mathrm{NC}_{2}\left(\mathrm{M}^{3+}\right)=\mathrm{x}$

Total $=3$

And if $\mathrm{I}_{\mathrm{i}}$ is an interaction parameter (i.e. bond strength) between the cation $\left(\mathrm{H}^{+}, \mathrm{M}^{2+}\right.$ or $\left.\mathrm{M}^{3+}\right)$ and the anion $\left(\mathrm{A}^{\mathrm{n}-}\right)$, a general model is given by:

$\mathrm{f}_{\mathrm{G}}(\mathrm{LDH})=\mathrm{p}_{0} \cdot \mathrm{f}_{0}(\mathrm{LDH})+\mathrm{p}_{1} \cdot \sum_{\mathrm{i}=1}^{3} \mathrm{w}_{\mathrm{i}} \cdot \mathrm{f}_{\mathrm{i}}(\mathrm{LDH})$

Where $\mathrm{p}_{0}$ might be thought as the probability of having interlayer anions that do not interact with the cations in the LDH layers. On the other hand, $\mathrm{p}_{1}$ is the probability of having interlayer anions interacting with the cations in the LDH layers. Therefore, $\mathrm{p}_{0}+\mathrm{p}_{1}=1$. And $\mathrm{w}$ is a weighing factor:

$\mathrm{w}_{\mathrm{i}}=\mathrm{NC}_{\mathrm{i}} \cdot \mathrm{I}_{\mathrm{i}} / \sum_{\mathrm{i}=1}^{3} \mathrm{NC}_{\mathrm{i}} \cdot \mathrm{I}_{\mathrm{i}}$

As a first approach to the simplification of this model, it is assumed that $\mathrm{p}_{0}=\mathrm{p}_{1}$ and also that the interaction between the cations in the layers and the anions in the interlayers has the same magnitu- de. Then, $I_{1}=I_{2}=I_{3}$, and $w_{1}=2 / 3, w_{2}=(1-x) / 3$ and $w_{3}=x / 3$. The standard Gibbs free energy of formation of LDHs as calculated by the general model is:

$$
\begin{aligned}
\Delta_{\mathrm{f}, \mathrm{GM}} \mathrm{G}_{\mathrm{m}}^{\mathrm{o}}\{\mathrm{T}, \mathrm{LDH}\} & =\mathrm{p}_{0} \cdot \Delta_{\mathrm{f}, \mathrm{M}_{0}} \mathrm{G}_{\mathrm{m}}^{\mathrm{o}}\{\mathrm{T}, \mathrm{LDH}\} \\
& +\mathrm{p}_{1} \cdot \sum_{\mathrm{i}=1}^{3} \mathrm{w}_{\mathrm{i}} \cdot \Delta_{\mathrm{f}, \mathrm{M}_{\mathrm{i}}} \mathrm{G}_{\mathrm{m}}^{\mathrm{o}}\{\mathrm{T}, \mathrm{LDH}\}
\end{aligned}
$$

Results of standard Gibbs free energies of formation for typical LDHs using the general model are shown in the last column of Table 1. Calculations are made using Equation 11. For example, the standard Gibbs free energies of formation for the $\left[\mathrm{Ni}_{0.75} \mathrm{Al}_{0.25}(\mathrm{OH})_{2}\right]\left(\mathrm{NO}_{3}\right)_{0.25}$ $\mathrm{LDH}$ calculated by the general model is:

$$
\begin{aligned}
\Delta_{\mathrm{f}, \mathrm{GM}} \mathrm{G}_{\mathrm{m}}^{\mathrm{o}} & \left\{298.15 \mathrm{~K}, \mathrm{NiAl}-\mathrm{NO}_{3}\right\}=\mathrm{p}_{0} \Delta_{\mathrm{f}, \mathrm{M} 0} \mathrm{G}_{\mathrm{m}}^{\mathrm{o}}\left\{298.15 \mathrm{~K}, \mathrm{NiAl}-\mathrm{NO}_{3}\right\} \\
& +\mathrm{p}_{1}\left[\mathrm{w}_{1} \cdot \Delta_{\mathrm{f}, \mathrm{M} 1} \mathrm{G}_{\mathrm{m}}^{\mathrm{o}}\left\{298.15 \mathrm{~K}, \mathrm{NiAl}-\mathrm{NO}_{3}\right\}\right. \\
& +\mathrm{w}_{2} \cdot \Delta_{\mathrm{f}, \mathrm{M} 2} \mathrm{G}_{\mathrm{m}}^{\circ}\left\{298.15 \mathrm{~K}, \mathrm{NiAl}-\mathrm{NO}_{3}\right\} \\
& \left.+\mathrm{w}_{3} \cdot \Delta_{\mathrm{f}, \mathrm{M} 3} \mathrm{G}_{\mathrm{m}}^{\mathrm{o}}\left\{298.15 \mathrm{~K}, \mathrm{NiAl}-\mathrm{NO}_{3}\right\}\right]
\end{aligned}
$$

$\Delta_{\mathrm{f}, \mathrm{GM}} \mathrm{G}_{\mathrm{m}}^{\mathrm{o}}\left\{298.15 \mathrm{~K}, \mathrm{NiAl}-\mathrm{NO}_{3}\right\}=0.5(-609.1)+0.5[(2 / 3)(-589.1)$

$$
+(0.75 / 3)(-594.9)-(0.25 / 3)(-589.1)=-599.8 \mathrm{~kJ} \mathrm{~mol}^{-1}
$$

The point about the general model is that it gives values for the thermodynamic properties of formation, which better represents the intrinsic stability of the hydrotalcites, as they take into account all the possible interactions of the interlayer anion and also do not depend on the use of an arbitrary starting material. Nevertheless, the quantities from the general model are weighed averages of the other models and the values are close to those given by the individual calculations.

\section{ANION EXCHANGE PROPERTIES OF LDH COMPOUNDS}

A general reaction for the anion exchange of $\mathrm{LDH}$ compounds is as follows:

$$
\begin{gathered}
{\left[\mathrm{M}_{1-\mathrm{x}}^{2+} \mathrm{M}_{\mathrm{x}}^{3+}(\mathrm{OH})_{2}\right]\left(\mathrm{A}^{\mathrm{n}-}\right)_{\mathrm{x} / \mathrm{n}}(\mathrm{s})+(\mathrm{x} / \mathrm{p}) \mathrm{B}^{\mathrm{p}-}(\mathrm{aq})=\left[\mathrm{M}_{1-\mathrm{x}}^{2+} \mathrm{M}_{\mathrm{x}}^{3+}(\mathrm{OH})_{2}\right] \mathrm{B}_{\mathrm{x} / \mathrm{p}}^{\mathrm{p}-}(\mathrm{s})} \\
+(\mathrm{x} / \mathrm{n}) \mathrm{A}^{\mathrm{n}-}(\mathrm{aq})
\end{gathered}
$$

If model 1 is used, then Equation 2 is replaced in Equation 12 to give:

$$
\begin{aligned}
(1-\mathrm{x}) \mathrm{M}^{2+}(\mathrm{OH})_{2}(\mathrm{~s})+(\mathrm{x}) \mathrm{M}^{3+}(\mathrm{OH})_{3}(\mathrm{~s})+(\mathrm{x} / \mathrm{n}) \mathrm{H}_{\mathrm{n}}\left(\mathrm{A}^{\mathrm{n}-}\right)(\mathrm{aq}) \\
+(\mathrm{x}) \mathrm{H}_{2} \mathrm{O}(\mathrm{aq})+(\mathrm{x} / \mathrm{p}) \mathrm{B}^{\mathrm{p}-}(\mathrm{aq})=(1-\mathrm{x}) \mathrm{M}^{2+}(\mathrm{OH})_{2}(\mathrm{~s}) \\
+(\mathrm{x}) \mathrm{M}^{3+}(\mathrm{OH})_{3}(\mathrm{~s})+(\mathrm{x} / \mathrm{p}) \mathrm{H}_{\mathrm{p}}\left(\mathrm{B}^{\mathrm{p}-}\right)(\mathrm{aq})+(\mathrm{x}) \mathrm{H}_{2} \mathrm{O}(\mathrm{aq}) \\
+(\mathrm{x} / \mathrm{n}) \mathrm{A}^{\mathrm{n}-}(\mathrm{aq})
\end{aligned}
$$

Adding $\mathrm{xOH}^{-}$to both sides of Equation 13 and rearranging terms, the standard Gibbs free energy change of anion exchange, $\Delta_{A E} G_{m}^{o}\{T$, $\mathrm{LDH}-\mathrm{A} / \mathrm{B}\}$, is given by:

$$
\begin{aligned}
\Delta_{\mathrm{AE}, \mathrm{M} 1} \mathrm{G}_{\mathrm{m}}^{\mathrm{o}} & \{\mathrm{T}, \mathrm{LDH}-\mathrm{A} / \mathrm{B}\}=(\mathrm{x})\left[(1 / \mathrm{p}) \Delta_{\mathrm{f}} \mathrm{G}_{\mathrm{m}}^{\mathrm{o}}\left\{\mathrm{T}, \mathrm{H}_{\mathrm{p}}\left(\mathrm{B}^{\mathrm{p}-}\right)\right\}\right. \\
& \left.-\Delta_{\mathrm{f}} \mathrm{G}_{\mathrm{m}}^{\mathrm{o}}\left\{\mathrm{T}, \mathrm{H}_{2} \mathrm{O}\right\}+\Delta_{\mathrm{f}} \mathrm{G}_{\mathrm{m}}^{\mathrm{o}}\left\{\mathrm{T}, \mathrm{OH}^{-}\right\}-(1 / \mathrm{p}) \Delta_{\mathrm{f}} \mathrm{G}_{\mathrm{m}}^{\mathrm{o}}\left\{\mathrm{T}, \mathrm{B}^{\mathrm{p}-}\right\}\right] \\
& -(\mathrm{x})\left[(1 / \mathrm{n}) \Delta_{\mathrm{f}} \mathrm{G}_{\mathrm{m}}^{\mathrm{o}}\left\{\mathrm{T}, \mathrm{H}_{\mathrm{n}}\left(\mathrm{A}^{\mathrm{n}-}\right)\right\}-\Delta_{\mathrm{f}} \mathrm{G}_{\mathrm{m}}^{\mathrm{o}}\left\{\mathrm{T}, \mathrm{H}_{2} \mathrm{O}\right\}\right. \\
& \left.\left.\left.+\Delta_{\mathrm{f}} \mathrm{G}_{\mathrm{m}}^{\mathrm{o}}\left\{\mathrm{T}, \mathrm{OH}^{-}\right)\right\}-(1 / \mathrm{n}) \Delta_{\mathrm{f}} \mathrm{G}_{\mathrm{m}}^{\mathrm{o}}\left\{\mathrm{T}, \mathrm{A}^{\mathrm{n}-}\right)\right\}\right]
\end{aligned}
$$


The terms in square brackets represent an anion contribution in the LDHs for model $1, \Delta_{\mathrm{CM} 1} \mathrm{G}_{\mathrm{m}}^{\circ}\{\mathrm{T}, \mathrm{LDH}-\mathrm{A}\}$.

Then,

$$
\begin{gathered}
\Delta_{\mathrm{CM} 1} \mathrm{G}_{\mathrm{m}}^{\mathrm{o}}\{\mathrm{T}, \mathrm{LDH}-\mathrm{A}\}=\Delta_{\mathrm{f}} \mathrm{G}_{\mathrm{m}}^{\mathrm{o}}\left\{\mathrm{T}, \mathrm{H}_{\mathrm{n}}\left(\mathrm{A}^{\mathrm{n}-}\right)\right\}-\Delta_{\mathrm{f}} \mathrm{G}_{\mathrm{m}}^{\mathrm{o}}\left\{\mathrm{T}, \mathrm{H}_{2} \mathrm{O}\right\} \\
\left.+\Delta_{\mathrm{f}} \mathrm{G}_{\mathrm{m}}^{\mathrm{o}}\left\{\mathrm{T}, \mathrm{OH}^{-}\right\}-(1 / \mathrm{n}) \Delta_{\mathrm{f}} \mathrm{G}_{\mathrm{m}}^{\mathrm{o}}\left\{\mathrm{T}, \mathrm{A}^{\mathrm{n}-}\right\}\right]
\end{gathered}
$$

The expression for $\Delta_{\mathrm{CM} 1} \mathrm{G}_{\mathrm{m}}^{\mathrm{o}}\{\mathrm{T}, \mathrm{LDH}-\mathrm{B}\}$ is similar to Equation 15 , where $\mathrm{A}^{\mathrm{n}-}$ is substituted by $\mathrm{B}^{\mathrm{m}-}$ and $\mathrm{n}$ by $\mathrm{p}$.

Finally,

$$
\begin{gathered}
\Delta_{\mathrm{AE}, \mathrm{M} 1} \mathrm{G}_{\mathrm{m}}^{\mathrm{o}}\{\mathrm{T}, \mathrm{LDH}-\mathrm{A} / \mathrm{B}\}=(\mathrm{x})\left[\Delta_{\mathrm{CM} 1} \mathrm{G}_{\mathrm{m}}^{\mathrm{o}}\{\mathrm{T}, \mathrm{LDH}-\mathrm{B}\}\right. \\
\left.-\Delta_{\mathrm{CM} 1} \mathrm{G}_{\mathrm{m}}^{\mathrm{o}}\{\mathrm{T}, \mathrm{LDH}-\mathrm{A}\}\right]
\end{gathered}
$$

Similar results are found for models 2 and 3. For model 0 , $\Delta_{\mathrm{AE}, \mathrm{M} 0} \mathrm{G}_{\mathrm{m}}^{\mathrm{o}}\{\mathrm{T}, \mathrm{LDH}-\mathrm{A}\}=0$ and, $\Delta_{\mathrm{CM} 0} \mathrm{G}_{\mathrm{m}}^{\mathrm{o}}\{\mathrm{T}, \mathrm{LDH}-\mathrm{A}\}=0$.

$$
\begin{gathered}
\Delta_{\mathrm{AE}, \mathrm{M} 2} \mathrm{G}_{\mathrm{m}}^{\mathrm{o}}\{\mathrm{T}, \mathrm{LDH}-\mathrm{A} / \mathrm{B}\}=(\mathrm{x})\left[\Delta_{\mathrm{CM} 2} \mathrm{G}_{\mathrm{m}}^{\mathrm{o}}\{\mathrm{T}, \mathrm{LDH}-\mathrm{B}\}\right. \\
\left.-\Delta_{\mathrm{CM} 2} \mathrm{G}_{\mathrm{m}}^{\mathrm{o}}\{\mathrm{T}, \mathrm{LDH}-\mathrm{A}\}\right]
\end{gathered}
$$

Where,

$$
\begin{aligned}
\Delta_{\mathrm{CM} 2} \mathrm{G}_{\mathrm{m}}^{\mathrm{o}} & \{\mathrm{T}, \mathrm{LDH}-\mathrm{A}\}=(1 / 2) \Delta_{\mathrm{f}} \mathrm{G}_{\mathrm{m}}^{\mathrm{o}}\left\{\mathrm{T}, \mathrm{M}^{2+}\left(\mathrm{A}^{\mathrm{n}-}\right)_{2 / \mathrm{n}}\right\} \\
& -(1 / 2) \Delta_{\mathrm{f}} \mathrm{G}_{\mathrm{m}}^{\circ}\left\{\mathrm{T}, \mathrm{M}^{2+}(\mathrm{OH})_{2}\right\}+\Delta_{\mathrm{f}} \mathrm{G}_{\mathrm{m}}^{\mathrm{o}}\left\{\mathrm{T}, \mathrm{OH}^{-}\right\} \\
& -(1 / \mathrm{n}) \Delta_{\mathrm{f}} \mathrm{G}_{\mathrm{m}}^{\mathrm{o}}\left\{\mathrm{T}, \mathrm{A}^{\mathrm{n}-}\right\}
\end{aligned}
$$

and

$$
\begin{gathered}
\Delta_{\mathrm{AE}, \mathrm{M} 3} \mathrm{G}_{\mathrm{m}}^{\mathrm{o}}\{\mathrm{T}, \mathrm{LDH}-\mathrm{A} / \mathrm{B}\}=(\mathrm{x})\left[\Delta_{\mathrm{CM} 3} \mathrm{G}_{\mathrm{m}}^{\mathrm{o}}\{\mathrm{T}, \mathrm{LDH}-\mathrm{B}\}\right. \\
\left.-\Delta_{\mathrm{CM} 3} \mathrm{G}_{\mathrm{m}}^{\mathrm{o}}\{\mathrm{T}, \mathrm{LDH}-\mathrm{A}\}\right]
\end{gathered}
$$

where,

$$
\begin{aligned}
\Delta_{\text {CM } 3} G_{m}^{\circ} & \{T, L D H-A\}=(1 / 3 n) \Delta_{\mathrm{f}} G_{m}^{o}\left\{T, M_{n}^{3+}\left(A^{\mathrm{n}-}\right)_{3}\right\} \\
& -(1 / 3) \Delta_{\mathrm{f}} \mathrm{G}_{\mathrm{m}}^{\circ}\left\{\mathrm{T}, \mathrm{M}^{3+}(\mathrm{OH})_{3}\right\}+\Delta_{\mathrm{f}} \mathrm{G}_{\mathrm{m}}^{\mathrm{o}}\left\{\mathrm{T}, \mathrm{OH}^{-}\right\} \\
& -(1 / \mathrm{n}) \Delta_{\mathrm{f}} \mathrm{G}_{\mathrm{m}}^{\mathrm{o}}\left\{\mathrm{T}, \mathrm{A}^{\mathrm{n}-}\right\}
\end{aligned}
$$
is:

The Gibbs free energy of anion exchange for the general model

$\Delta_{\mathrm{AE}, \mathrm{GM}} \mathrm{G}_{\mathrm{m}}^{\mathrm{o}}\{\mathrm{T}, \mathrm{LDH}-\mathrm{A} / \mathrm{B}\}=(\mathrm{x})\left[\Delta_{\mathrm{CGM}} \mathrm{G}_{\mathrm{m}}^{\mathrm{o}}\{\mathrm{T}, \mathrm{LDH}-\mathrm{B}\}\right.$

$$
\left.-\Delta_{\mathrm{CGM}} \mathrm{G}_{\mathrm{m}}^{\mathrm{o}}\{\mathrm{T}, \mathrm{LDH}-\mathrm{A}\}\right]
$$

where $\Delta_{\mathrm{CGM}} \mathrm{G}_{\mathrm{m}}^{\mathrm{o}}\{\mathrm{LDH}-\mathrm{A}\}$ and $\Delta_{\mathrm{CGM}} \mathrm{G}_{\mathrm{m}}^{\mathrm{o}}\{\mathrm{LDH}-\mathrm{B}\}$ have the following form:

$$
\begin{aligned}
\Delta_{\mathrm{CGM}} \mathrm{G}_{\mathrm{m}}^{\mathrm{o}}\{\mathrm{LDH}-\mathrm{A}\} & =\mathrm{p}_{0} \cdot \Delta_{\mathrm{CM} 0} \mathrm{G}_{\mathrm{m}}^{\mathrm{o}}\{\mathrm{T}, \mathrm{LDH}-\mathrm{A}\} \\
& +\mathrm{p}_{1} \cdot \sum_{\mathrm{i}=1}^{3} \mathrm{w}_{\mathrm{i}} \cdot \Delta_{\mathrm{CMi}} \mathrm{G}_{\mathrm{m}}^{\mathrm{o}}\{\mathrm{T}, \mathrm{LDH}-\mathrm{A}\}
\end{aligned}
$$

Calculations of standard Gibbs free energy anion contribution terms for models 1, 2 and 3 are made by Equations 15, 18 and 20, respectively. For instance, the standard Gibbs free energy anion contribution term for the $\left[\mathrm{Cu}_{1-\mathrm{x}}^{2+} \mathrm{M}_{\mathrm{x}}^{3+}(\mathrm{OH})_{2}\right] \mathrm{Cl}_{\mathrm{x}} \mathrm{LDHs}$ calculated by model 2 is:

$$
\begin{aligned}
\Delta_{\mathrm{CM} 2} \mathrm{G}_{\mathrm{m}}^{\circ} & \left\{298.15 \mathrm{~K}, \mathrm{CuM}^{3+}-\mathrm{Cl}\right\}=(1 / 2) \Delta_{\mathrm{f}} \mathrm{G}_{\mathrm{m}}^{\mathrm{o}}\left\{298.15 \mathrm{~K}, \mathrm{CuCl}_{2}\right\} \\
& -(1 / 2) \Delta_{\mathrm{f}} \mathrm{G}_{\mathrm{m}}^{\mathrm{o}}\left\{298.15 \mathrm{~K}, \mathrm{Cu}(\mathrm{OH})_{2}\right\} \\
& +\Delta_{\mathrm{f}} \mathrm{G}_{\mathrm{m}}^{\mathrm{o}}\left\{298.15 \mathrm{~K}, \mathrm{OH}^{-}\right\}-\Delta_{\mathrm{f}} \mathrm{G}_{\mathrm{m}}^{\circ}\left\{298.15 \mathrm{~K}, \mathrm{Cl}^{-}\right\} \\
\Delta_{\mathrm{CM} 2} \mathrm{G}_{\mathrm{m}}^{\circ} & \left\{298.15 \mathrm{~K}, \mathrm{CuM}^{3+}-\mathrm{Cl}\right\}=(1 / 2)(-171.8)-(1 / 2)(-357.7) \\
+ & (-157.3)-(-131.3)=66.9 \mathrm{~kJ} \mathrm{~mol}^{-1}
\end{aligned}
$$

Other results for models 1,2 and 3 are calculated in a similar manner. They are shown in Tables 3, 4 and 5, respectively. The thermodynamic data of the compounds were taken from the compilations already mentioned ${ }^{22-24}$.

Examples of standard Gibbs free energies of anion exchange for some LDHs, calculated for the different models, are shown in Table 6. Calculations were made using Equations 16, 17, 19 and 22, and data from Tables 3, 4 and 5 were taken. For example, the standard Gibbs free energy change of the $\mathrm{F}^{-}$anion exchange for the $\left[\mathrm{Zn}_{0.66} \mathrm{Al}_{0.33}(\mathrm{OH})_{2}\right](\mathrm{Cl})_{0.33} \mathrm{LDH}$ as calculated by model 2 is:

$\Delta_{\mathrm{AE},{ }_{\mathrm{M} 2}} \mathrm{G}_{\mathrm{m}}^{\mathrm{o}}\{298.15 \mathrm{~K}, \mathrm{ZnAl}-\mathrm{Cl} / \mathrm{F}\}=(\mathrm{x})\left[\Delta_{\mathrm{CM} 2} \mathrm{G}_{\mathrm{m}}^{\mathrm{o}}\{298.15 \mathrm{~K}, \mathrm{ZnAl}-\mathrm{F}\}\right.$
$\left.-\Delta_{\mathrm{CM} 2} \mathrm{G}_{\mathrm{m}}^{\mathrm{o}}\{298.15 \mathrm{~K}, \mathrm{ZnAl}-\mathrm{Cl}\}\right]$

$\Delta_{\mathrm{AE}, \mathrm{M} 2} \mathrm{G}_{\mathrm{m}}^{\mathrm{o}}\{298.15 \mathrm{~K}, \mathrm{ZnAl}-\mathrm{Cl} / \mathrm{F}\}=0.33[43.2-65.4]=-7.4 \mathrm{~kJ} \mathrm{~mol}^{-1}$

Other results shown in Table 6 are calculated in a similar manner.

Table 3. Standard Gibbs free energy anion contribution term for Model 1, $\Delta_{\text {CM1 }} \mathrm{G}_{\mathrm{m}}^{\circ}\{298.15 \mathrm{~K}, \mathrm{LDH}-\mathrm{A}\}$

$$
\mathrm{A}^{\mathrm{n}-}, \Delta_{\mathrm{CM} 1} \mathrm{G}_{\mathrm{m}}^{\mathrm{o}}\{298.15 \mathrm{~K}, \mathrm{LDH}-\mathrm{A}\}\left(\mathrm{kJ} \mathrm{mol}^{-1}\right)
$$

\begin{tabular}{lcccccc} 
Cation & $\mathrm{I}^{-}$ & $\mathrm{Br}^{-}$ & $\mathrm{Cl}^{-}$ & $\mathrm{NO}_{3}^{-}$ & $\mathrm{F}^{-}$ & $\mathrm{OH}^{-}$ \\
\hline $\mathrm{H}$ & 79.9 & 79.9 & 79.9 & 80.0 & 63.0 & 0 \\
\hline
\end{tabular}

Table 4. Standard Gibbs free energy anion contribution term for Model 2, $\Delta_{\mathrm{CM} 2} \mathrm{G}_{\mathrm{m}}^{\circ}\{298.15 \mathrm{~K}, \mathrm{LDH}-\mathrm{A}\}$

\begin{tabular}{lrrrrrr}
\hline & \multicolumn{5}{c}{$\mathrm{A}^{\mathrm{n}-}, \Delta_{\mathrm{CM} 2} \mathrm{G}_{\mathrm{m}}^{\circ}\{298.15 \mathrm{~K}, \mathrm{LDH}-\mathrm{A}\}\left(\mathrm{kJ} \mathrm{mol}^{-1}\right)$} \\
$\mathrm{M}^{2+}$ & \multicolumn{1}{c}{$\mathrm{I}^{-}$} & \multicolumn{1}{c}{$\mathrm{Br}^{-}$} & \multicolumn{1}{c}{$\mathrm{Cl}^{-}$} & \multicolumn{1}{c}{$\mathrm{NO}_{3}^{-}$} & \multicolumn{1}{c}{$\mathrm{F}^{-}$} & $\mathrm{OH}^{-}$ \\
\hline $\mathrm{Ba}$ & 22.3 & 9.5 & 2.7 & -15.8 & -20.8 & 0 \\
$\mathrm{Be}$ & 197.3 & 179.0 & 160.4 & 0.0 & 48.7 & 0 \\
$\mathrm{Ca}$ & 75.9 & 34.6 & 46.3 & 31.6 & -16.8 & 0 \\
$\mathrm{Cd}$ & 29.6 & 36.3 & 39.1 & 59.4 & 34.4 & 0 \\
$\mathrm{Co}$ & 73.4 & 69.5 & 64.8 & 66.7 & 44.0 & 0 \\
$\mathrm{Cu}$ & 61.2 & 73.6 & 66.9 & 71.9 & 58.9 & 0 \\
$\mathrm{Fe}$ & 71.7 & 71.6 & 64.1 & 43.2 & 50.9 & 0 \\
$\mathrm{Hg}$ & -8.2 & 13.5 & 32.3 & 69.1 & 89.5 & 0 \\
$\mathrm{Mg}$ & 131.2 & 114.1 & 94.9 & 76.7 & 3.9 & 0 \\
$\mathrm{Mn}$ & 61.6 & 63.3 & 53.1 & 5.4 & 46.8 & 0 \\
$\mathrm{Ni}$ & 70.7 & 61.6 & 65.3 & 57.1 & 36.7 & 0 \\
$\mathrm{~Pb}$ & 21.3 & 30.9 & 30.8 & 41.8 & 26.7 & 0 \\
$\mathrm{Pd}$ & -5.1 & 45.9 & 44.3 & -68.0 & 20.9 & 0 \\
$\mathrm{Sn}$ & 68.1 & 68.1 & 89.9 & -51.8 & 33.4 & 0 \\
$\mathrm{Sr}$ & 48.3 & 27.8 & 13.5 & -5.9 & -22.5 & 0 \\
$\mathrm{Zn}$ & 67.1 & 67.9 & 65.4 & 81.9 & 43.2 & 0 \\
\hline
\end{tabular}

\section{DISCUSSION}

\section{LDH thermodynamic properties of formation}

Results of the standard Gibbs free energies of formation for typical LDHs, calculated by the single and the general models, are shown in Table 1. It can be seen that similar results are obtained for all the single models. The general model results from the weighed combination of the single models. Therefore, the standard Gibbs free energies of formation as calculated by the general model are expected to be close to the values predicted by the single models. From the $\mathrm{Mg}-\mathrm{Al}, \mathrm{Ni}-\mathrm{Al}$ and $\mathrm{Zn}-\mathrm{Al} \mathrm{LDH}$ examples in Table 1, deviations of the thermodynamic quantities obtained by the single models 0,1 , and 2 from the general model can be as large as $12 \mathrm{~kJ} \mathrm{~mol}^{-1}$ for 
Table 5. Standard Gibbs free energy anion contribution term for Model 3, $\Delta_{\mathrm{CM} 3} \mathrm{G}_{\mathrm{m}}{ }^{\circ}\{298.15 \mathrm{~K}, \mathrm{LDH}-\mathrm{A}\}$

\begin{tabular}{lrrrrrr}
\hline \multicolumn{5}{c}{$\left.\mathrm{A}^{\mathrm{n}-}, \Delta_{\mathrm{CM}_{3} \mathrm{G}_{\mathrm{m}}^{\mathrm{o}}\{298.15} \mathrm{K}, \mathrm{LDH}-\mathrm{A}\right\}\left(\mathrm{kJ} \mathrm{mol}^{-1}\right)$} \\
$\mathrm{M}^{3+}$ & \multicolumn{1}{c}{$\mathrm{I}^{-}$} & \multicolumn{1}{c}{$\mathrm{Br}^{-}$} & $\mathrm{Cl}^{-}$ & $\mathrm{NO}_{3}^{-}$ & $\mathrm{F}^{-}$ & $\mathrm{OH}^{-}$ \\
\hline $\mathrm{Al}$ & 176.2 & 169.7 & 113.7 & 80.1 & 35.5 & 0 \\
$\mathrm{Au}$ & 26.8 & 55.0 & 74.1 & $\mathrm{x}$ & 139.9 & 0 \\
$\mathrm{Bi}$ & 30.1 & 41.3 & 63.2 & $\mathrm{x}$ & 10.0 & 0 \\
$\mathrm{Ce}$ & 89.7 & 53.6 & 57.5 & $\mathrm{x}$ & -37.7 & 0 \\
$\mathrm{Cr}$ & 109.1 & 58.1 & 59.9 & $\mathrm{x}$ & 57.3 & 0 \\
$\mathrm{Dy}$ & 120.5 & 79.7 & 93.2 & $\mathrm{x}$ & $\mathrm{x}$ & 0 \\
$\mathrm{Er}$ & 131.0 & 92.3 & 109.4 & $\mathrm{x}$ & $\mathrm{x}$ & 0 \\
$\mathrm{Eu}$ & 52.3 & 35.0 & 46.6 & $\mathrm{x}$ & $\mathrm{x}$ & 0 \\
$\mathrm{Fe}$ & 92.2 & 103.0 & 100.0 & 78.9 & 24.0 & 0 \\
$\mathrm{Ga}$ & 100.4 & 107.7 & 88.3 & $\mathrm{x}$ & 87.6 & 0 \\
$\mathrm{Gd}$ & 119.8 & 75.0 & 93.2 & $\mathrm{x}$ & $\mathrm{x}$ & 0 \\
$\mathrm{In}$ & 107.1 & 83.6 & 79.7 & $\mathrm{x}$ & 62.0 & 0 \\
$\mathrm{La}$ & 91.4 & 75.8 & 58.9 & $\mathrm{x}$ & -38.0 & 0 \\
$\mathrm{Lu}$ & 131.4 & 104.5 & 100.7 & $\mathrm{x}$ & $\mathrm{x}$ & 0 \\
$\mathrm{Nd}$ & 98.9 & 63.1 & 68.8 & $\mathrm{x}$ & $\mathrm{x}$ & 0 \\
$\mathrm{Pr}$ & 102.1 & 56.6 & 70.2 & $\mathrm{x}$ & -4.6 & 0 \\
$\mathrm{Pu}$ & 98.8 & 83.3 & 66.3 & $\mathrm{x}$ & 15.6 & 0 \\
$\mathrm{Sb}$ & 87.0 & 91.3 & 90.2 & $\mathrm{x}$ & 68.2 & 0 \\
$\mathrm{Sc}$ & 109.5 & 122.0 & 101.3 & $\mathrm{x}$ & 12.0 & 0 \\
$\mathrm{Sm}$ & 118.8 & 69.5 & 78.7 & $\mathrm{x}$ & $\mathrm{x}$ & 0 \\
$\mathrm{~Tb}$ & 93.2 & 75.9 & 88.3 & $\mathrm{x}$ & $\mathrm{x}$ & 0 \\
$\mathrm{Tl}$ & 2.7 & 39.5 & 45.9 & $\mathrm{x}$ & 120.6 & 0 \\
$\mathrm{Tm}$ & 125.5 & 85.2 & 100.5 & $\mathrm{x}$ & $\mathrm{x}$ & 0 \\
$\mathrm{Y}$ & 149.8 & 187.9 & 106.6 & $\mathrm{x}$ & 6.5 & 0 \\
$\mathrm{Yb}$ & 148.5 & 90.6 & 112.6 & $\mathrm{x}$ & $\mathrm{x}$ & 0 \\
\hline $\mathrm{x}$ & & & & & & \\
\hline
\end{tabular}

$\mathrm{x}=$ not enough data available chloride and nitrate LDHs. Model 3 deviates the most from the predicted values by the general model. Deviations can be as large as $18 \mathrm{~kJ} \mathrm{~mol}^{-1}$ for chloride LDHs and $10 \mathrm{~kJ} \mathrm{~mol}^{-1}$ for nitrate LDHs. The observed differences can be explained by the variability of the published thermodynamic data of the single compounds and by the restrictions imposed by the mixture models ${ }^{19}$.

Standard Gibbs free energies of formation for several green rustlike compounds have been recently compiled ${ }^{30}$. Green rust is a layered double hydroxide with the approximate composition $\left[\mathrm{Fe}_{4}^{2+} \mathrm{Fe}_{2}^{3+}(\mathrm{OH})_{12}\right] \mathrm{SO}_{4} \cdot 3 \mathrm{H}_{2} \mathrm{O}$. For example, Hansen et al. ${ }^{31}$ calculated the standard free energy of formation of $\left[\mathrm{Fe}_{0.67} \mathrm{Fe}_{0.33}(\mathrm{OH})_{2}\right]\left(\mathrm{SO}_{4}\right)_{0.17}$ to be $-611.3 \mathrm{~kJ} \mathrm{~mol}^{-1}$ by solubility methods, Refait et al. ${ }^{32}$ reported the value $-631.7 \mathrm{~kJ} \mathrm{~mol}^{-1}$ using redox potential methods and recently Hansen ${ }^{30}$ reported a new value of $-631.8 \mathrm{~kJ} \mathrm{~mol}^{-1}$ using solubility methods. As seen from these free energy results, variability among published thermodynamic data can be found. From the results in Table 1, models 1 and 2 give the best predictions for the free energy value of $-611.3 \mathrm{~kJ} \mathrm{~mol}^{-1}$, with absolute errors of about $5 \mathrm{~kJ} \mathrm{~mol}^{-1}$. On the other hand, models 0 and 3 agree quite well with the free energy value $-631.7 \mathrm{~kJ} \mathrm{~mol}^{-1}$. Absolute errors of about $1 \mathrm{~kJ} \mathrm{~mol}^{-1}$ are obtained for both models. The general model predicts an intermediate free energy value of $-621.8 \mathrm{~kJ} \mathrm{~mol}^{-1}$, with absolute deviations of about 10 $\mathrm{kJ} \mathrm{mol}^{-1}$ from both experimental free energy values.

The standard Gibbs free energy of formation of $\left[\mathrm{Fe}_{0.75} \mathrm{Fe}_{0.25}(\mathrm{OH})_{2}\right](\mathrm{Cl})_{0.25}$ as calculated by redox potential methods is $-536.5 \mathrm{~kJ} \mathrm{~mol}^{-133}$. This result is in good agreement with the predicted values by model $0\left(-535.2 \mathrm{~kJ} \mathrm{~mol}^{-1}\right)$ and the general model $(-525.5 \mathrm{~kJ}$ $\left.\mathrm{mol}^{-1}\right)$, as seen from the results in Table 1 . Moreover, predictions by models 1, 2 and 3 have absolute errors of about $25 \mathrm{~kJ} \mathrm{~mol}^{-1}$. Genin et $a l .{ }^{33}$ reported the value of the standard Gibbs free energy of formation of $-598.3 \mathrm{~kJ} \mathrm{~mol}^{-1}$ for $\left[\mathrm{Fe}_{0.67} \mathrm{Fe}_{0.33}(\mathrm{OH})_{2}\right]\left(\mathrm{CO}_{3}\right)_{0.17}$ as calculated by redox potential methods. From results in Table 1, models 0 and 2 agree the best, with absolute errors of 2 and $6 \mathrm{~kJ} \mathrm{~mol}^{-1}$, respectively.

Table 6. Standard Gibbs free energy change of the anion exchange for some chloride LDHs using different models

\begin{tabular}{|c|c|c|c|c|c|c|c|}
\hline \multirow[b]{2}{*}{ LDH-A } & \multirow[b]{2}{*}{ Model } & \multicolumn{6}{|c|}{$\mathrm{B}^{\mathrm{p}-}, \Delta_{\mathrm{AE}} \mathrm{G}_{\mathrm{m}}^{\mathrm{o}}\{298.15 \mathrm{~K}, \mathrm{LDH}-\mathrm{Cl} / \mathrm{B}\}\left(\mathrm{kJ} \mathrm{mol}^{-1}\right)$} \\
\hline & & $\mathrm{I}^{-}$ & $\mathrm{Br}^{-}$ & $\mathrm{Cl}^{-}$ & $\mathrm{NO}_{3}^{-}$ & $\mathrm{F}^{-}$ & $\mathrm{OH}^{-}$ \\
\hline \multirow{4}{*}[\mathrm{Zn}_{0.67}\mathrm{Al}_{0.33}(\mathrm{OH})_{2}]{$(\mathrm{Cl})_{0.33}$} & 1 & 0.0 & 0.0 & 0.0 & 0.0 & -5.6 & -26.6 \\
\hline & 2 & 0.6 & 0.8 & 0.0 & 5.5 & -7.4 & -21.8 \\
\hline & 3 & 20.8 & 18.7 & 0.0 & -11.2 & -26.1 & -37.9 \\
\hline & General & 1.0 & 0.9 & 0.0 & 0.3 & -3.9 & -13.2 \\
\hline \multirow{4}{*}[\mathrm{Mg}_{0.70}\mathrm{Al}_{0.30}(\mathrm{OH})_{2}]{$(\mathrm{Cl})_{0.30}$} & 1 & 0.0 & 0.0 & 0.0 & 0.0 & -5.1 & -24.0 \\
\hline & 2 & 10.9 & 5.8 & 0.0 & -5.5 & -27.3 & -28.5 \\
\hline & 3 & 18.7 & 16.8 & 0.0 & -10.1 & -23.5 & -34.1 \\
\hline & General & 2.2 & 1.4 & 0.0 & -1.1 & -6.1 & -13.0 \\
\hline \multirow{4}{*}[\mathrm{Cu}_{0.50}\mathrm{Al}_{0.50}(\mathrm{OH})_{2}]{$(\mathrm{Cl})_{0.50}$} & 1 & 0.0 & 0.0 & 0.0 & 0.0 & -8.4 & -40.0 \\
\hline & 2 & -2.8 & 3.3 & 0.0 & 2.5 & -4.0 & -33.5 \\
\hline & 3 & 31.2 & 28.0 & 0.0 & -16.8 & -39.1 & -56.9 \\
\hline & General & 1.0 & 1.6 & 0.0 & -0.4 & -5.0 & -19.9 \\
\hline \multirow{4}{*}[\mathrm{Ni}_{0.75}\mathrm{Al}_{0.25}(\mathrm{OH})_{2}]{$(\mathrm{Cl})_{0.25}$} & 1 & 0.0 & 0.0 & 0.0 & 0.0 & -4.2 & -20.0 \\
\hline & 2 & 1.3 & -0.9 & 0.0 & -2.1 & -7.1 & -16.3 \\
\hline & 3 & 15.6 & 14.0 & 0.0 & -8.4 & -19.6 & -28.4 \\
\hline & General & 0.8 & 0.5 & 0.0 & -0.6 & -3.1 & -9.9 \\
\hline
\end{tabular}


Allada et al. ${ }^{19}$ measured by calorimetric methods the enthalpies of formation of $\left[\mathrm{Co}_{0.68} \mathrm{Al}_{0.32}(\mathrm{OH})_{2}\right]\left(\mathrm{CO}_{3}\right)_{0.17} \cdot 0.80 \mathrm{H}_{2} \mathrm{O}$ and $\left[\mathrm{Co}_{0.76} \mathrm{Al}_{0.24}(\mathrm{OH})_{2}\right]\left(\mathrm{CO}_{3}\right)_{0.12} \cdot 0.81 \mathrm{H}_{2} \mathrm{O} \mathrm{LDHs}$, obtaining values of 1044.2 and $-991.8 \mathrm{~kJ} \mathrm{~mol}^{-1}$, respectively. As shown in Table 2, predicted values by models 0 and 2 agree quite well with the latter values, where model 0 deviates the least $\left(3\right.$ and $8 \mathrm{~kJ} \mathrm{~mol}^{-1}$, respectively).

\section{Anion exhange properties of LDH compounds}

From the thermodynamic study of the anion exchange of LDHs, a simple anion contribution term, $\Delta_{\mathrm{C}} \mathrm{G}_{\mathrm{m}}^{\mathrm{o}}\{298.15 \mathrm{~K}, \mathrm{LDH}-\mathrm{A}\}$, is obtained. This contribution term together with Equations 16, 17 and 19 for the single models and Equation 21 for the general model can be used to estimate anion exchange selectivities of LDHs. A compilation of $\Delta_{\mathrm{C}} \mathrm{G}_{\mathrm{m}}^{\mathrm{o}}\{298.15 \mathrm{~K}, \mathrm{LDH}-\mathrm{A}\}$ values for the single models is given in Tables 3, 4 and 5. As seen from Equations 15, 18 and 20, the results for $\mathrm{OH}^{-}$contributions should be null. This is a direct result of the utilization of models based on the mixture of metal hydroxides. Results of standard Gibbs free energy change of the anion exchange for several LDHs, as calculated by all the mixture models, are given in Table 6. If we take into account that the larger the negative value of the free energy the more probable the anion exchange, then anion selectivities can be easily obtained. So far, very few papers have been published dealing with detailed thermodynamic studies of the anion exchange for LDHs. For example, Israëli et al. ${ }^{34}$ carried out a microcalorimetric study of the anion exchange for a $\left[\mathrm{Zn}_{0.67} \mathrm{Al}_{0.33}(\mathrm{OH})_{2}\right](\mathrm{Cl})_{0.33} \mathrm{LDH}$. They obtained the following order of selectivity based on the free energies values: $\mathrm{OH}^{-}\left(\Delta_{\mathrm{AE}} \mathrm{G}_{\mathrm{m}}^{\mathrm{o}}\{298.15 \mathrm{~K}, \mathrm{ZnAl}-\mathrm{Cl} / \mathrm{OH}\}=-10 \mathrm{~kJ} \mathrm{~mol}^{-1}\right)>\mathrm{Cl}^{-}$ $\left(\Delta_{\mathrm{AE}} \mathrm{G}_{\mathrm{m}}^{\circ}\{298.15 \mathrm{~K}, \mathrm{ZnAl}-\mathrm{Cl} / \mathrm{Cl}\}=0 \mathrm{~kJ} \mathrm{~mol}^{-1}\right)>\mathrm{NO}_{3}^{-}\left(\Delta_{\mathrm{AE}} \mathrm{G}_{\mathrm{m}}^{\mathrm{o}}\{298.15\right.$ $\left.\left.\mathrm{K}, \mathrm{ZnAl}-\mathrm{Cl} / \mathrm{NO}_{3}\right\}=3.6 \mathrm{~kJ} \mathrm{~mol}^{-1}\right)$. Even though the errors associated with the predicted LDH thermodynamic quantities can be as large as $10 \mathrm{~kJ} \mathrm{~mol}^{-1}$, the standard Gibbs free energy change of anion exchange calculated by the models agrees reasonable well with the experimental findings and predicts the same order of $\mathrm{LDH}$ selectivity: $\mathrm{OH}^{-}$ $\left(\Delta_{\mathrm{AE}, \mathrm{M} 2} \mathrm{G}_{\mathrm{m}}^{\circ}\{298.15 \mathrm{~K}, \mathrm{ZnAl}-\mathrm{Cl} / \mathrm{OH}\}=-21.8 \mathrm{~kJ} \mathrm{~mol}^{-1}\right)>\mathrm{Cl}^{-}$ $\left(\Delta_{\mathrm{AE}} \mathrm{G}_{\mathrm{m}}^{\mathrm{o}}\{298.15 \mathrm{~K}, \mathrm{ZnAl}-\mathrm{Cl} / \mathrm{Cl}\}=0 \mathrm{~kJ} \mathrm{~mol}^{-1}\right)>\mathrm{NO}_{3}^{-}\left(\Delta_{\mathrm{AE}, \mathrm{M} 2} \mathrm{G}_{\mathrm{m}}^{\mathrm{o}}\{298.15\right.$ $\left.\mathrm{K}, \mathrm{ZnAl}-\mathrm{Cl} / \mathrm{NO}_{3}\right\}=5.5 \mathrm{~kJ} \mathrm{~mol}^{-1}$ ) for model 2 and $\mathrm{OH}$ $\left(\Delta_{\mathrm{AE}, \mathrm{GM}} \mathrm{G}_{\mathrm{m}}^{\mathrm{o}}\{298.15 \mathrm{~K}, \mathrm{ZnAl}-\mathrm{Cl} / \mathrm{OH}\}=-13.2 \mathrm{~kJ} \mathrm{~mol}^{-1}\right)>\mathrm{Cl}$ $\left(\Delta_{\mathrm{AE}}^{\mathrm{AE}} \mathrm{G}_{\mathrm{m}}^{\circ}\{298.15 \mathrm{~K}, \mathrm{ZnAl}-\mathrm{Cl} / \mathrm{Cl}\}=0 \mathrm{~kJ} \mathrm{~mol}^{-1}\right)>\mathrm{NO}_{3}$ $\left(\Delta_{\mathrm{AE}, \mathrm{GM}} \mathrm{G}_{\mathrm{m}}^{\mathrm{o}}\left\{298.15 \mathrm{~K}, \mathrm{ZnAl}-\mathrm{Cl} / \mathrm{NO}_{3}\right\}=0.3 \mathrm{~kJ} \mathrm{~mol}^{-1}\right)$ for the general model. From the anion exchange free energy results for the $\mathrm{Zn}-\mathrm{Al}$ $\mathrm{LDH}$, model 2 and the general model seem to work better than the other models. In general terms, all models predict a small standard Gibbs free energy change of anion exchange for the LDHs. This result conforms with published data for $\mathrm{LDHs}^{34}$ and anion styrenic resins ${ }^{35}$. Miyata $^{5}$ studied the anion exchange properties of a $\left[\mathrm{Mg}_{0.70} \mathrm{Al}_{0.30}(\mathrm{OH})_{2}\right]\left(\mathrm{NO}_{3}\right)_{0.30} \mathrm{LDH}$. He found the following order of anion selectivity for monovalent anions: $\mathrm{OH}^{-}>\mathrm{F}^{-}>\mathrm{Cl}^{-}>\mathrm{Br}^{-}>\mathrm{NO}_{3}^{-}>$ $\mathrm{I}^{-}$. The selectivity order predicted by all the models is: $\mathrm{OH}^{-}>\mathrm{F}^{-}>\mathrm{NO}_{3}-$ $>\mathrm{Cl}^{-}>\mathrm{Br}^{-}>\mathrm{I}^{-}$which agrees quite well with the order given by Miyata ${ }^{5}$. For the results in Table 6, the chloride LDH is taken as the basis for the thermodynamic calculations for the ease of comparison of the free energy changes of the LDH anion exchange. Nevertheless, the final order predicted for the selectivity would be the same regardless of the LDH taken as the basis for the calculations. With the exception of Israëli et al.'s work ${ }^{34}$, all other studies do not provide enough LDH quantitative thermodynamic data. Yamaoka et al. ${ }^{36}$ investigated the anion exchange properties of a $\left[\mathrm{Cu}_{0.50} \mathrm{Al}_{0.50}(\mathrm{OH})_{2}\right]\left(\mathrm{CO}_{3}\right)_{0.25} \mathrm{LDH}$. For monovalent anions the following order of selectivity is found: $\mathrm{F}^{-}>\mathrm{Cl}^{-}$ $>\mathrm{I}^{-}>\mathrm{NO}_{3}$. The order of selectivities predicted by all the models are: model $1, \mathrm{~F}^{-}>\mathrm{Cl}^{-} \sim \mathrm{I}^{-} \sim \mathrm{NO}_{3}^{-}$; model $2, \mathrm{~F}^{-}>\mathrm{I}^{-}>\mathrm{Cl}^{-}>\mathrm{NO}_{3}^{-}$; model 3, $\mathrm{F}^{-}$ $>\mathrm{NO}_{3}^{-}>\mathrm{Cl}^{-}>\mathrm{I}^{-}$; general model, $\mathrm{F}^{-}>\mathrm{NO}_{3}^{-}>\mathrm{Cl}^{-}>\mathrm{I}^{-}$. In this case, model 1 seems to agree better with the experimental results than the other models. We should not forget that the real anion exchange phenomenon involves several other variables that influence the anion exchange selectivity and are not considered in this thermodynamic study. Some of these variables include the water content and base strength of the $\mathrm{LDH}$, the concentration, size and charge of the counterions and the nature of the solvent $t^{37-39}$. Aditionally, the anion exchange selectivity predictions are based on the reference states of $298.15 \mathrm{~K}$ and $101325 \mathrm{~Pa}$ for the compounds and a hypothetical ideal $1 \mathrm{~m}\left(\mathrm{~mol} \mathrm{~kg}^{-1}\right)$ solution at $298.15 \mathrm{~K}$ and $101325 \mathrm{~Pa}$ for the dissolved species. Bish ${ }^{40}$ studied the anion exchange in the mineral takovite, a layered double hydroxide with the general formula $\mathrm{Ni}_{6} \mathrm{Al}_{2}(\mathrm{OH})_{16} \mathrm{CO}_{3} \cdot 4 \mathrm{H}_{2} \mathrm{O}$. He proposed the following order of preference for monovalent anions: $\mathrm{NO}_{3}^{-}>\mathrm{OH}^{-} \sim$ $\mathrm{Cl}^{-}$. He assumed $\mathrm{OH}^{-}$to behave similarly to $\mathrm{Cl}^{-}$. From the results in Table 6, the predicted order is in all cases: $\mathrm{OH}^{-}>\mathrm{NO}_{3}{ }^{-}>\mathrm{Cl}^{-}$. This order is in agreement with the experimental selectivity order $\mathrm{NO}_{3}^{-}>\mathrm{Cl}^{-}$. However, if the published experimental results for $\mathrm{LDH}$ anion exchange and the $\mathrm{OH}^{-}$standard Gibbs free energy change, as given in Table 6 , are considered, we may say that Bish's assumption of $\mathrm{OH}^{-} \sim \mathrm{Cl}^{-}$is not correct, and the $\mathrm{OH}^{-}$correct order should be the one predicted by the models $\mathrm{OH}^{-}>\mathrm{NO}_{3}^{-}>\mathrm{Cl}^{-}$.

In the thermodynamic study of the synthesis of LDH from metal oxides by hydrothermal-reconstruction (H-R) methods, we recently found that the anion contribution term for model 2 can be used to predict the order of selectivity for anion LDH reconstruction ${ }^{20}$. The free energy change of reaction for the synthesis of LDH by H-R methods as calculated by model 2 is related to the free energy of hydration of the metal oxides and the free energy anion contribution term by the following ${ }^{20}$ :

$$
\begin{aligned}
& \Delta_{\mathrm{HR}, \mathrm{M} 2} \mathrm{G}_{\mathrm{m}}^{\mathrm{o}}\{\mathrm{T}, \mathrm{LDH}-\mathrm{A}\}=(1-\mathrm{x}) \Delta_{\mathrm{H}} \mathrm{G}_{\mathrm{m}}^{\mathrm{o}}\left\{\mathrm{T}, \mathrm{M}^{2+} \mathrm{O}\right\} \\
& +(\mathrm{x}) \Delta_{\mathrm{H}} \mathrm{G}_{\mathrm{m}}^{\mathrm{o}}\left\{\mathrm{T}, \mathrm{M}_{2}^{3+} \mathrm{O}_{3}\right\}+(\mathrm{x}) \Delta_{\mathrm{CM} 2} \mathrm{G}_{\mathrm{m}}^{\mathrm{o}}\{\mathrm{T}, \mathrm{LDH}-\mathrm{A}\} .
\end{aligned}
$$

This is an interesting result since the anion contribution term is also directly related to the anion exchange selectivity of LDHs, as shown by Equations 16, 17, 19 and 22. A direct advantage of this finding is that experimental anion exchange selectivities of LDHs may be used to estimate the selectivity for anion sorption on mixed oxides or vice versa. This procedure is supported by Parker et al.'s results $^{41}$. They found the following order of sorption of monovalent anions for a mixture of $\mathrm{Mg}$ - $\mathrm{Al}$ oxides (obtained from the calcination of a $\left.\mathrm{Mg}_{6} \mathrm{Al}_{2}(\mathrm{OH})_{16} \mathrm{CO}_{3} \cdot 4 \mathrm{H}_{2} \mathrm{O} \mathrm{LDH}\right): \mathrm{F}^{-}>\mathrm{Cl}^{-}>\mathrm{NO}_{3}$. This order is the same order of preference of anion exchange for a $\left[\mathrm{Mg}_{0.70} \mathrm{Al}_{0.30}(\mathrm{OH})_{2}\right]\left(\mathrm{NO}_{3}\right)_{0.30} \mathrm{LDH}$ as reported by Miyata ${ }^{5}$.

\section{CONCLUSION}

In this work, four new single mixture models and one general model for the estimation of thermodynamic properties of LDHs are presented. The single models $0,1,2$ and 3 give similar results when predicting standard Gibbs free energies of formation for LDHs. However, model 2 and the general model agree the best with reported experimental values. For the calculation of enthalpies of formation for LDHs, models 0 and 2 conform quite well with published data. The utilization of these models can contribute to a better understanding of the synthesis and physicochemical properties of LDH materials which are of interest not only in academic research, but also in technological applications.

In spite of the scarcity of anion exchange data for LDHs, some agreement is found between experimental and predicted thermodynamic quantities obtained using the models. Although anion exchange selectivity depends on a diverse number of factors and the actual order of preference can be only accurately determined by 
experimentation $^{39}$, the results presented in this work may be of great use when experimental results are not available. Likewise, results in Tables 3, 4 and 5 are intended to serve only as a guide for prediction of anion exchange selectivity for LDHs. A quantitative thermodynamic analysis of the anion exchange on LDHs is expected to be difficult because the error range of the predictions by the mixture models can be of the same order of magnitude of the thermodynamic quantities in the anion exchange. However, good quantitative agreement is found for the standard free energy change of anion exchange for a $\mathrm{Zn}-\mathrm{Al} \mathrm{LDH}$. More experimental results are required in order to select the best mixture model. So far, model 2 and the general model seem to give the best results. Model 2 has the advantage of simplicity, requiring less thermodynamic information than the general model, but the general model has the attractiveness of being less arbitrary and averaging more information, when available.

Anion exchange selectivities for LDHs are found to be related to anion sorption order of preference for mixed metal oxides derived or related to LDHs. This result is shown by comparison of the standard Gibbs free energy anion contribution term in both processes.

The order of anion preference shown by the LDHs might be useful for the selection of precursors for the synthesis of new LDH materials by anion exchange methods ${ }^{42}$. The anion selectivity for $\mathrm{Mg}-\mathrm{Al} \mathrm{LDHs}^{5}$ : $\mathrm{OH}^{-}>\mathrm{F}^{-}>\mathrm{Cl}^{-}>\mathrm{Br}^{-}>\mathrm{NO}_{3}^{-}>\mathrm{I}^{-}$indicates that $\mathrm{I}^{-}$and $\mathrm{NO}_{3}^{-}$are the most easily exchangeable anions in the $\mathrm{Mg}-\mathrm{Al} \mathrm{LDH}$. Therefore, in the synthesis of new intercalated LDHs by anion exchange methods, $\mathrm{Mg}-\mathrm{Al}-\mathrm{I}$ and $\mathrm{Mg}-\mathrm{Al}-\mathrm{NO}_{3} \mathrm{LDHs}$ can be the best precursors. These $\mathrm{I}^{-}$ and $\mathrm{NO}_{3}$ - interlayer anions in $\mathrm{Mg}$-Al LDHs are also thermodynamically favored to be exchanged for other anions.

\section{ACKNOWLEDGMENTS}

We acknowledge financial support for this work by the Director, Division of Chemical and Thermal Systems of the National Science Foundation under grant CTS-0321979 and from Universidad Industrial de Santander and COLCIENCIAS, in the frame of the project "Synthesis, characterization and testing of biomimetic catalysts for selective oxidation", code 1102-05665-95.

\section{AUXILIARY TABLES}

Table 7. Standard Gibbs free energies of formation for some species of $\mathrm{A}^{-}, \mathrm{HA}$ and $\mathrm{M}^{2+}\left(\mathrm{A}^{-}\right)_{2}^{22-24}$

\begin{tabular}{lrrrrrr}
\hline & \multicolumn{7}{c}{$\Delta_{\mathrm{f}} \mathrm{G}_{\mathrm{m}}^{\mathrm{o}}\{298.15 \mathrm{~K}\}$} \\
& $\mathrm{I}^{-}$ & $\mathrm{Br}^{-}$ & $\mathrm{Cl}^{-}$ & $\mathrm{NO}_{3}^{-}$ & $\mathrm{F}^{-}$ & $\mathrm{OH}^{-}$ \\
\hline $\mathrm{A}^{-}(\mathrm{aq})$ & -51.6 & -104.2 & -131.3 & -111.4 & -280.0 & -157.3 \\
$\mathrm{HA}(\mathrm{aq})$ & -51.6 & -104.0 & -131.3 & -111.3 & -296.9 & -237.2 \\
$\mathrm{Ba}^{2+}$ & -598.7 & -729.7 & -797.3 & -794.7 & -1141.8 & -854.8 \\
$\mathrm{Be}^{2+}$ & -212.5 & -354.4 & -445.7 & -726.8 & -966.5 & -818.5 \\
$\mathrm{Ca}^{2+}$ & -533.7 & -721.7 & -752.3 & -742.2 & -1176.0 & -897.0 \\
$\mathrm{Cd}^{2+}$ & -203.8 & -295.8 & -344.2 & -264.0 & -651.1 & -474.5 \\
$\mathrm{Co}^{2+}$ & -97.5 & -210.5 & -274.1 & -230.5 & -613.0 & -455.6 \\
$\mathrm{Cu}^{2+}$ & -23.8 & -104.4 & -171.8 & -122.2 & -485.3 & -357.7 \\
$\mathrm{Fe}^{2+}$ & -129.3 & -234.7 & -303.8 & -305.9 & -627.6 & -484.1 \\
$\mathrm{Hg}^{2+}$ & -100.4 & -162.3 & -178.7 & -65.5 & -361.8 & -295.4 \\
$\mathrm{Mg}^{2+}$ & -359.8 & -499.2 & -591.8 & -588.5 & -1071.1 & -833.6 \\
$\mathrm{Mn}^{2+}$ & -264.2 & -365.9 & -440.5 & -496.2 & -750.6 & -598.7 \\
$\mathrm{Ni}^{2+}$ & -89.1 & -212.5 & -259.2 & -236.0 & -613.8 & -441.8 \\
$\mathrm{~Pb}^{2+}$ & -173.5 & -259.7 & -314.0 & -252.3 & -619.7 & -427.6 \\
$\mathrm{Pd}^{2+}$ & -90.1 & -93.3 & -150.6 & -335.6 & -494.9 & -291.3 \\
$\mathrm{Sn}^{2+}$ & -143.9 & -249.4 & -259.7 & -503.5 & -670.3 & -491.6 \\
$\mathrm{Sr}^{2+}$ & -562.3 & -708.5 & -791.2 & -790.4 & -1160.6 & -870.3 \\
$\mathrm{Zn}^{2+}$ & -208.9 & -312.6 & -371.5 & -298.8 & -713.4 & -554.5 \\
\hline
\end{tabular}

Table 8. Standard Gibbs free energies of formation for some species of $\mathrm{M}^{3+}\left(\mathrm{A}^{-}\right)_{3}^{22-24}$

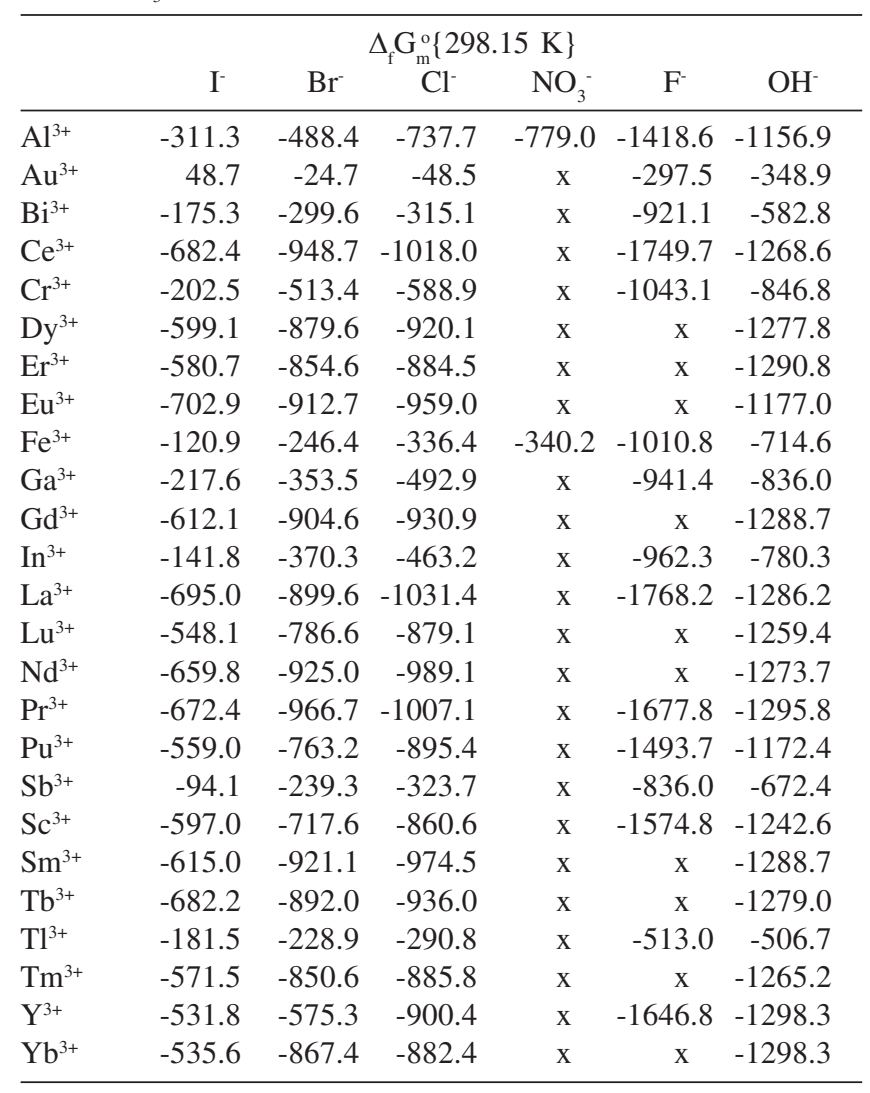

$\mathrm{x}=$ data not available

Table 9. Standard Gibbs free energies of formation for some species of $\mathrm{A}^{2-}, \mathrm{H}_{2}\left(\mathrm{~A}^{2-}\right)$ and $\mathrm{M}^{2+}\left(\mathrm{A}^{2-}\right)^{22-24}$

\begin{tabular}{lc}
\hline Specie & $\Delta_{\mathrm{f}} \mathrm{G}_{\mathrm{m}}^{\circ}\{298.15 \mathrm{~K}\}, \mathrm{kJ} \mathrm{mol}^{-1}$ \\
\hline $\mathrm{CO}_{3}^{2-}$ & -528.0 \\
$\mathrm{SO}_{4}^{2-}$ & -743.9 \\
$\mathrm{H}_{2} \mathrm{CO}_{3}(\mathrm{aq})$ & -623.0 \\
$\mathrm{H}_{2} \mathrm{SO}_{4}(\mathrm{aq})$ & -744.8 \\
$\mathrm{FeCO}_{3}$ & -674.0 \\
$\mathrm{FeSO}_{4}$ & -818.0 \\
$\mathrm{Fe}_{2}\left(\mathrm{SO}_{4}\right)_{3}$ & -2681.5 \\
\hline
\end{tabular}

Table 10. Standard enthalpies of formation for some species ${ }^{19,22-24}$

\begin{tabular}{lc}
\hline Specie & $\Delta_{\mathrm{f}} \mathrm{H}_{\mathrm{m}}^{\circ}\{298.15 \mathrm{~K}\}, \mathrm{kJ} \mathrm{mol}^{-1}$ \\
\hline $\mathrm{OH}^{-}(\mathrm{aq})$ & -230.0 \\
$\mathrm{CO}_{3}^{2-}(\mathrm{aq})$ & -677.1 \\
$\mathrm{H}_{2} \mathrm{O}^{(\mathrm{l})}$ & -285.8 \\
$\mathrm{H}_{2} \mathrm{CO}_{3}(\mathrm{aq})$ & -699.6 \\
$\mathrm{Co}(\mathrm{OH})_{2}$ & -544.7 \\
$\mathrm{Al}(\mathrm{OH})_{3}$ & -1293.3 \\
$\mathrm{CoCO}_{3}$ & -745.8 \\
\hline
\end{tabular}

\section{REFERENCES}

1. Cavani, F.; Trifiro, F.; Vaccari, A.; Catal. Today 1991, 11, 173.

2. Sels, B. F.; de Vos, D. E.; Jacobs, P. A.; Catal. Rev. Sci. Eng. 2001, 43, 443. 
3. Rives, V.; Ulibarri, M. A.; Coord. Chem. Rev. 1999, 181, 61.

4. Châtelel, L.; Bottero, J. Y.; Yvon, J.; Bouchelaghem, A.; Colloids Surf., A 1996, 111, 167.

5. Miyata, S.; Clays Clay Miner. 1983, 31, 305

6. Camino, G.; Maffezzoli, A.; Braglia, M.; De Lazzaro, M.; Zammarano, M.; Polym. Degrad. Stab. 2001, 74, 457.

7. van der Ven, L.; van Gemert, M. L. M.; Batenburg, L. F.; Keern, J. J.; Gielgens, L. H.; Koster, T. P. M.; Fischer, H. R.; Appl. Clay Sci. 2000, 17, 25

8. Vatier, J.; Ramdani, A.; Vitre, M. T.; Mignon, M.; Arzneim.-Forsch. 1994, 44, 514.

9. Gardner, E.; Huntoon, K. M.; Pinnavaia, T. J.; Adv. Mater. 2001, 13, 1263

10. Dutta, P. K.; Puri, M.; J. Phys. Chem. 1989, 93, 376.

11. Buchheit, R. G.; Mamidipally, S. B.; Schmutz, P.; Guan, H.; Corrosion (Houston, TX, U.S.) 2002, 58, 3.

12. Morigi, M.; Scavetta, E.; Berrettoni, M.; Giorgetti, M.; Tonelli, D.; Anal. Chim. Acta 2001, 439, 265.

13. Hussein, M. Z. B.; Zainal, Z.; Yahaya, A.; Foo, D. W. V.; J. Controlled Release 2002, 82, 417.

14. Ambrogi, V.; Fardella, G.; Grandollini, G.; Perioli, L.; Int. J. Pharm. 2001, $220,23$.

15. Trifiro, F.; Vaccari, A. In Comprehensive Supramolecular Chemistry; Atwood, J. L.; Davies, J. E. D.; MacNicol, D. D.; Vogtle, F.; Lehn, J. M.; Alberti, G.; Bein, T., eds.; Pergamon: Oxford, 1996; vol. 7, p. 251.

16. Hofmeister, W.; von Platen, H.; Cryst. Rev. 1992, 3, 3.

17. Allman, R.; Acta Crystallogr., Sect. B: Struct. Sci. 1968, 24, 972.

18. Rives, V.; Layered Double hydroxides: Present and Future, Nova Science publishers, Inc: New York, 2001.

19. Allada, R. K.; Navrotsky, A.; Berbeco, H. T.; Casey, W. H.; Science 2002, 296, 721.

20. Bravo-Suárez, J. J.; Páez-Mozo, E. A.; Oyama, S. T.; Quim. Nova 2004, $27,601$.

21. Bookin, A. S.; Drits, V. A.; Clays Clay Miner. 1993, 41, 551.

22. Naumov, G. B.; Ryzhenko, B. N.; Khodakovsky, I. L.; Handbook of Thermodynamic Data, U.S. Geological Survey: Washington, D.C., 1974.
23. Karapet'yants, M. Kh.; Karapet'yants, M. L.; Thermodynamic Constants of Inorganic and Organic Compounds, Ann Arbor-Humphrey Science Publishers: Ann Arbor, MI, 1970.

24. Perry, H. R.; Green, D. W.; Maloney, J. O.; Perry's Chemical Engineers' Handbook, McGraw-Hill: New York, 1997.

25. Newman, S. P.; Greenwell, H. C.; Coveney, P. V.; Jones, W. In ref. 18, p. 93.

26. Fogg, A. M.; Rohl, A. L.; Parkinson, G. M.; O’hare, D.; Chem. Mater. 1999, $11,1194$.

27. Kalinichev, A. G.; Kirkpatrick, R. J.; Cygan, R. T.; Am. Mineral. 2000, 85, 1046.

28. Hou, X.; Kalinichev, A. G.; Kirkpatrick, R. J.; Chem. Mater. 2002, 14, 2078.

29. Kalinichev, A. G.; Kirkpatrick, R. J.; Chem. Mater. 2002, 14, 3539.

30. Hansen, H. C. B. In ref. 18, p. 413.

31. Hansen, H. C. B.; Borggaard, O. K.; Sørensen, J.; Geochim. Cosmochim. Acta 1994, 58, 2599.

32. Refait, Ph.; Bon, C.; Simon, L.; Bourrié, G.; Trolard, F.; Bessière, J.; Génin, J.-M. R.; Clay Miner. 1999, 34, 499.

33. Génin, J.-M. R.; Bourrié, G.; Trolard, F.; Abdelmoula, M.; Jaffrezic, A.; Refait, P.; Maitre, V.; Humbert, B.; Herbillon, A.; Environ. Sci. Technol. 1998, 32, 1058.

34. Israëli, Y.; Taviot-Guého, C.; Besse, J. P.; Morel, J. P.; Morel-Desrosiers, N.; J. Chem. Soc., Dalton Trans. 2000, 791.

35. Harland, C. E.; Ion Exchange: Theory and Practice, The Royal Society of Chemistry: Cambridge, UK, 1994.

36. Yamaoka, T.; Abe, M.; Tsuji, M.; Mater. Res. Bull. 1989, 24, 1183.

37. Reichenberg, D. In Ion Exchange; Marinsky, J. A., ed.; Marcel Dekker, Inc.: New York, 1966, p. 227

38. Diamond, R. M. In ref. 37, p. 277.

39. Marinsky, J. A. In ref. 37, p. 353.

40. Bish, D. L.; Bull. Minéral. 1980, 103, 170.

41. Parker, L. M.; Milestone, N. B.; Newman, R. H.; Ind. Eng. Chem. Res. 1995, 34, 1196.

42. de Roy, A.; Forano, C.; Besse, J. P. In ref. 18, p. 1. 